\title{
Improving P2P Live-Content Delivery using SVC
}

\author{
T. Schierl ${ }^{\mathrm{a}}$, Y. Sánchez ${ }^{\mathrm{b}}$, C. Hellge ${ }^{\mathrm{a}, \mathrm{b}}$, and T. Wiegand ${ }^{\mathrm{a}, \mathrm{b}}$ \\ ${ }^{a}$ Fraunhofer HHI, Image Processing Department, Germany \\ ${ }^{\mathrm{b}}$ Technische Universität Berlin, Department of Telecommunication Systems, Germany
}

\begin{abstract}
P2P content delivery techniques for video transmission have become of high interest in the last years. With the involvement of client into the delivery process, P2P approaches can significantly reduce the load and cost on servers, especially for popular services. However, previous studies have already pointed out the unreliability of P2P-based live streaming approaches due to peer churn, where peers may ungracefully leave the P2P infrastructure, typically an overlay networks. Peers ungracefully leaving the system cause connection losses in the overlay, which require repair operations. During such repair operations, which typically take a few roundtrip times, no data is received from the lost connection. While taking low delay for fast-channel tune-in into account as a key feature for broadcast-like streaming applications, the P2P live streaming approach can only rely on a certain media pre-buffer during such repair operations. In this paper, multi-tree based Application Layer Multicast as a P2P overlay technique for live streaming is considered. The use of Flow Forwarding (FF), a.k.a. Retransmission, or Forward Error Correction (FEC) in combination with Scalable video Coding (SVC) for concealment during overlay repair operations is shown. Furthermore the benefits of using SVC over the use of AVC single layer transmission are presented.
\end{abstract}

Keywords: P2P Content Delivery, ALM, SVC, Live streaming, Multi-tree, Error control, Internet Broadcast

\section{INTRODUCTION}

In the last few years P2P content delivery infrastructures have become a new option for providing live video content over the Internet. The lack of IP Multicast [1] deployment and the high investments required for Content Delivery Networks (CDNs) for content delivery over the Internet have lead to an increasing interest in P2P architectures, also for live video systems.

Overlay Multicast, in particular Application Layer Multicast (ALM), may significantly reduce the resources required by CDNs and may be the only way to allow Multicast-like delivery over the Internet. The requirements for ALM on customer equipment with respect to additional processing power and memory are expected to be small, when compared to the existing capabilities, e.g. of IPTV set-top boxes, especially for live content delivery.

In order to make ALM an appealing approach for live content delivery and to compete with traditional broadcasting services, ALM has to fulfill two main constraints, which are a low channel switching time, i.e. typically half a second in digital TV services, and a reasonable video quality. Low switching times can, e.g., be obtained if using tree-based Overlay Multicast structures in case of P2P, which implies the use of a push-based media delivery between nodes [2]. Contrary to that, the pull-based mesh distribution approach is an alternative P2P technique that typically introduces higher delays due to the overlay construction and data delivery, as discussed in [3].

While releasing the server from the heavy burden of delivering the live video content to a multitude of users, live content delivery based on P2P is inherently unreliable, if peers cannot be controlled by a central instance or Quality-of-Service provision is missing for the connection of a peer to and via the Internet. Although multi-tree based ALM [4][5] is a more error-robust variant of ALM, the video transmission may still be disrupted due to peer churn, especially due to peers leaving the network ungracefully, nodes losing connectivity or because of temporal bandwidth variations caused by higher-priority user tasks running on peers [4]. Due to these potential disruptions in the media transmission, which result in connection outages, error control methods need to be applied in order to meet the real time requirements of live transmissions. 
The main focus of this work is to improve the media transport under real time requirements in the presence of frequent connection outages in multi-tree based ALM environments. The approach presented in this work is to use error control techniques in combination with SVC to overcome such outages. These techniques are prioritized Flow Forwarding and unequal Forward Error Correction.

The remainder of the paper is organized as follows. In section 2, the multi-tree ALM is described. Section 2.1 introduces the basic ideas on SVC, whereas the applied error concealment methods in combination with SVC are explained in section 3. In section 4 the performed simulations and obtained results are presented. The paper is concluded in section 5 .

\section{MULTIPLE-TREE ALM using SVC}

In order to meet competitive switching times as provided by today's digital TV broadcast systems, the most promising P2P architecture is thought to be the tree-based Application Layer Multicast (ALM) approach. In such an approach, there is a central entity, here called Application Layer Tree Manager (ALTM), which deals with the tree maintenance. This entity is responsible for integrating joining nodes into the tree, as well as for "repairing" the overlay due to leaving nodes. The ALTM has to cope with peer churn, which implies many peers joining and leaving the overlay within a short period of time. Peers joining the service should not result in any disturbance of the media delivery for already connected users if well addressed. But the ALTM need to be able to integrate all the nodes in a short period of time to achieve fast switching times. However, peers ungracefully leaving the overlay lead to a partitioned overlay, where peers depending on such leaving nodes may not receive all media packets.

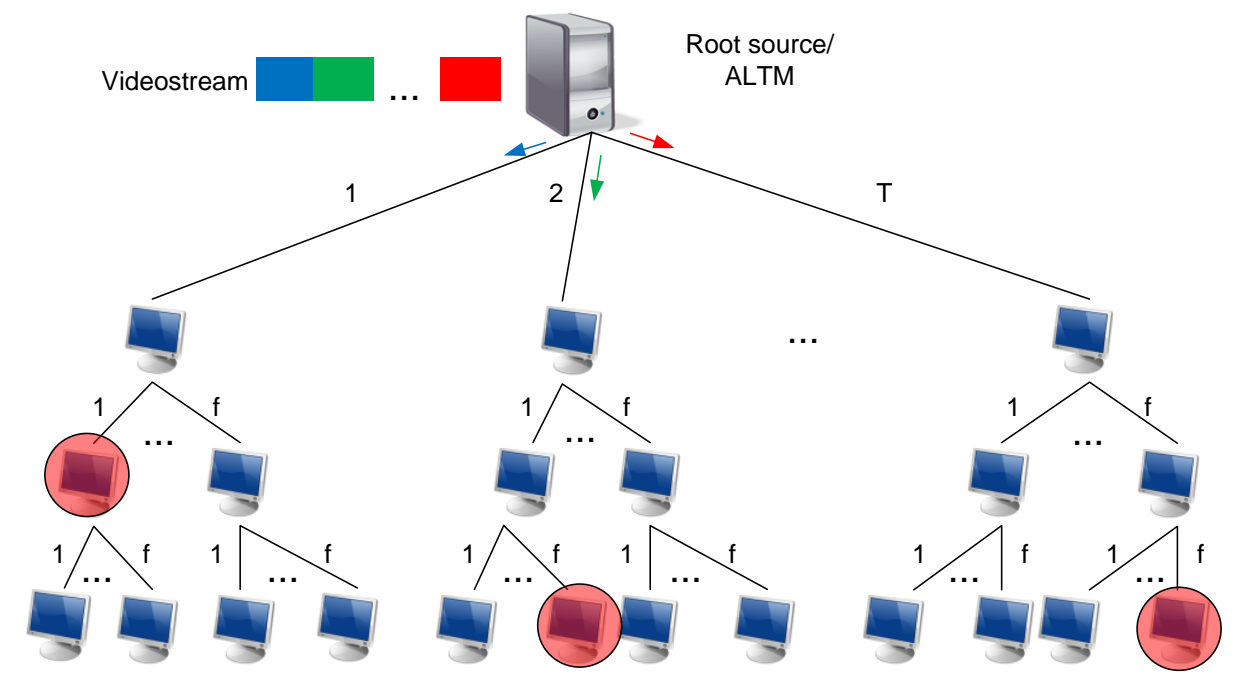

Figure 1- Multi-tree Application Layer Multicast (a single peer is marked in different trees)

The multiple tree approach [4] and [5] is a special form of the tree-based ALM approach. In this case, the streaming root source divides the video data into different parts and sends each part over a different tree, as shown in Figure 1. Peers have fixed relationships imposed by the ALTM, in which they instantaneously forward the received messages to their dependent peers, minimizing the latency of the system. A node receives the data from all the trees but is only a serving or parent node in a single tree, i.e. this node is an inner node in the tree, and at the same time it is a leaf peer in all the other trees, where it is served by other relay or parent nodes. Thus, a higher robustness is obtained through source diversity, where a disconnection from a tree means the loss of only part of the video content instead of the loss of all the data as in single-tree approaches.

\subsection{SCALABLE VIDEO CODING}

In order to improve the delivery of video data within each tree, a layered video codec such as Scalable Video Coding (SVC) [6] may further allow for improvement and flexibility in transport during outages and transmission rate variation. SVC allows for dropping parts of the media stream in order to reduce the 
transmission rate while still achieving a reasonable playout quality at the receiver in terms of frame rate, picture quality and resolution. The main idea is to overcome outages in the overlay trees during live transmission by displaying the video at reduced quality, which is controlled by the SVC codec. After an outage, the system resumes the playout at the client with full quality.

The approach of using SVC in ALM has been already presented in [7], where it is used for supporting heterogeneous devices and coping with congestion between overlay peers. In this work, we extend the use of SVC in order to cope with outages during overlay repair operations.

\section{ERROR CONTROL FOR OVERLAY OUTAGES}

As already mentioned, live transmission over ALM may suffer from overlay outages. In order to overcome these outage periods where a client cannot receive all the required media data, two different methods are compared in this work:

I. Flow Forwarding (FF) or Retransmission.

II. Forward Error Correction (FEC).

While point-to-point systems can perform prioritized retransmission or adjust the FEC when packet losses occur, multi-tree $\mathrm{P} 2 \mathrm{P}$ overlays have some additional difficulties. That is, when carrying out a prioritized retransmission, contrary to point-to-point approaches, the peer requested for retransmission may not have the missing packets available, due to splitting the stream into multiple trees. Additionally, FEC approaches, which are adaptively applied based on changing reception situations of nodes, become more difficult, since the adaptive FEC may be applied at the source, thus typically all peers are affected by such a FEC adjustment at the source.

Due to these issues in multi-tree ALM, the media delivery with SVC presents an adequate alternative in this type of P2P system, since its layered quality representation in combination with an unequal treatment of the SVC media layers in error control allows for graceful degradation in case of peers ungracefully leaving the system and causing delivery disruptions.

\subsection{Flow Forwarding with SVC}

The Automatic Repeat reQuest (ARQ) approach provides resending of packets after loss detection [8]. Such loss detection is carried out using incremental sequence numbers within the transmitted packets in order to detect losses. When complete path outages occur, a client may request multiple missing packets for retransmission from other sources, if are available. Typically the ARQ mechanism is limited by a constrained uplink bandwidth at peers, which may not allow sending all packets requested for retransmission.

In order to allow retransmission within RTP, the RTP payload format for retransmission [9] in combination with the Extended RTCP Feedback [10] can be used, where each RTP flow contains an SSRC-multiplexed retransmission session. The SVC NAL unit header's priority indicator field [6] can be used to ensure that the more important packets, typically the base layer packets, are transmitted first. The remaining available bandwidth is then assigned to the retransmission requests of the less important packets, e.g. enhancement layer packets. This process is referred to as Prioritized Retransmission. 


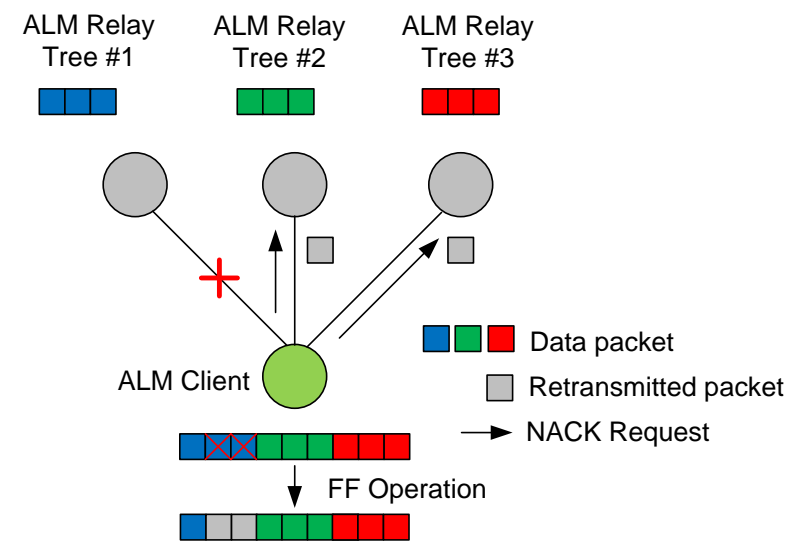

Figure 2- Flow Forwarding (Retransmission) in ALM to overcome the disconnection from Tree\#1

After detecting packet loss, a node requests the lost packets as well as the packets assumed to be lost in future via RTCP NAK messages from one or more still connected serving parent peers, the ALM Relay nodes. In order to share the additional bandwidth required for the retransmission, the requests for the missing packet-flow of the lost tree is equally distributed over the connected trees as also applied in [7] and shown in Figure 2.

Retransmission requests cost an additional time, which has to be taken into account to allow low switching times. Therefore, maximum end-to-end playout delay is limited and consequently the number of possible retransmission requests and transmissions too. This delay comprises the so called worst-case-delay $\left(t_{w c d}\right)$, the decoding delay and the retransmission delay $\left(t_{\text {ret }}\right)$, as shown in Figure 3.

- The worst case delay occurs along the slowest path in the overlay network and is defined as the time elapsed from when the packet is sent by the root source until the packet is received by the target receiver [11].

- A second type of delay, the decoding delay consists of the time needed to receive the media data that allows for decoding pictures (SVC dec delay), i.e. all the reference frames of the SVC Group of Pictures (GOP) [6].

- Finally, the retransmission delay corresponds to the time needed to request a given number of retransmission requests ( $n \_r e t$ in Figure 3). For each retransmission request a time equal to twice the one way delay (tow_delay) is needed. The tow_delay is shown in eq. (1), where $L$ may be the retransmitted or the retransmission request packet size, $T_{p}$ is the propagation delay in the link and $B$ is the bandwidth of the peers, which needs to be higher than the video rate $R$.

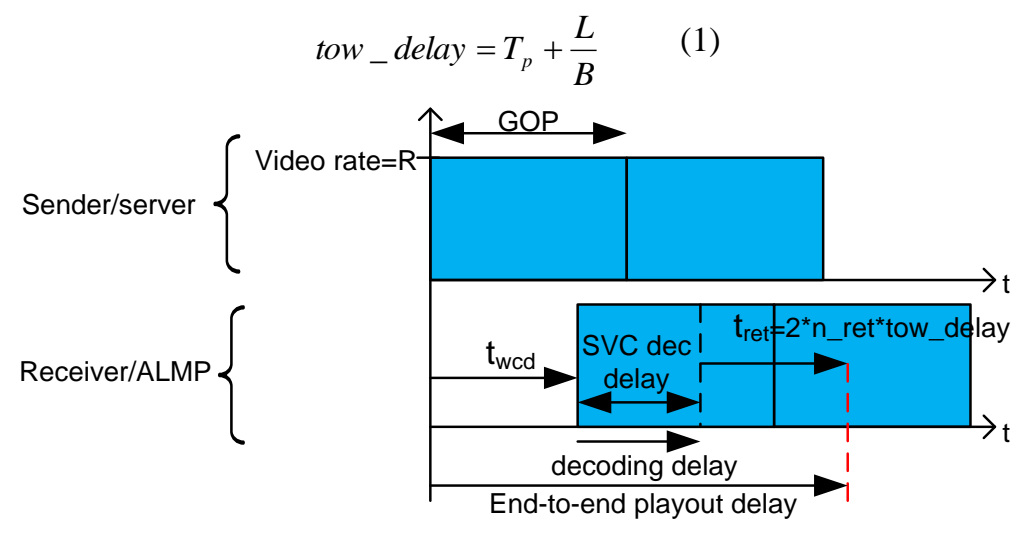

Figure 3: End-to-end playout delay for the FF case

\subsection{FEC Coding with SVC}

Forward error correction (FEC) technologies are typically used for reducing losses on multicast or broadcast channels without feedback channel. Redundancy symbols are added to the media stream, which can be further used to correct errors by using a specific algorithm. In Figure 4, an illustration of a layered FEC protection 
scheme, which allows for unequal protection, is depicted using multiple trees. A potential symbol distribution scheme to $T$ trees is shown in Figure 5, where $k_{x}$ are the source symbols (systematic symbols) and $n_{x}$ are all coded symbols for layer $x$ with $n_{x}-k_{x}$ redundant symbols. The code rate is defined as $k / n$.

Using FEC in multi-tree ALM requires a continuous redundancy overhead in all trees as shown in Figure 4. In case of temporarily losing a tree connection (Tree \#1 in the figure), the redundant data received from the other trees allows for recovering the lost data after FEC decoding.

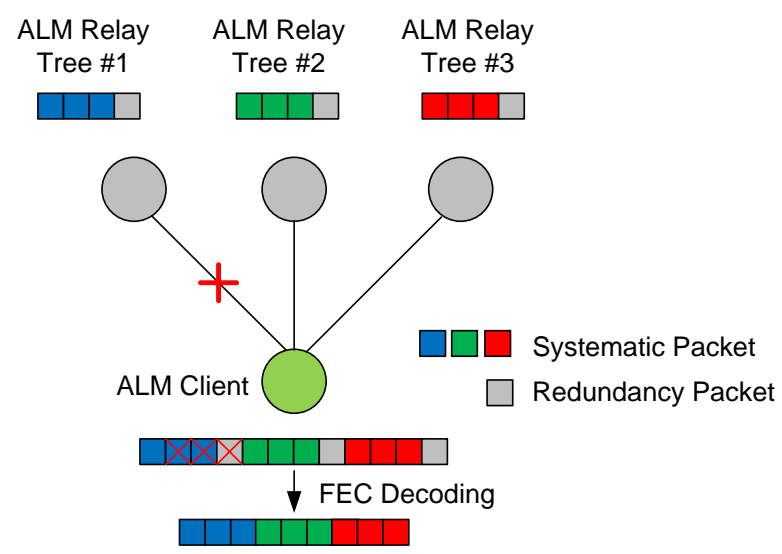

Figure 4 - Forward Error Correction in multi-tree based ALM to overcome the disconnection from Tree\#1

If each layer is transmitted in a separate RTP session, assignment of different FEC code rates may be possible, denoted as Unequal Error Protection. The IETF FEC framework [12] may be used to apply FEC to the different layers transported in separate RTP sessions, as shown in Figure 5.

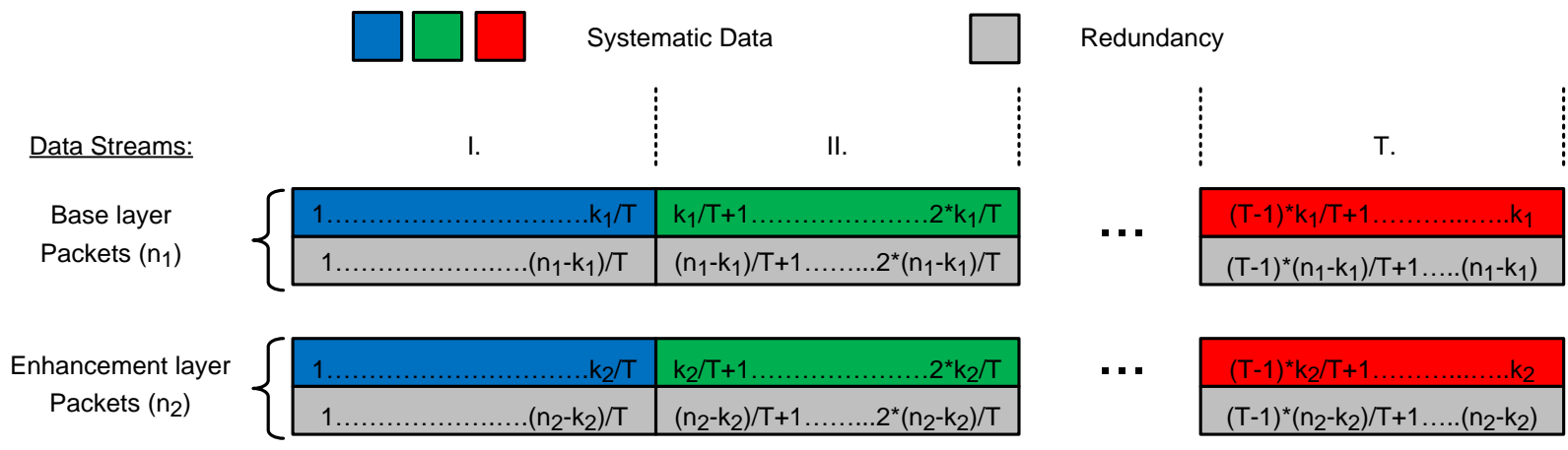

Figure 5- FEC structure for delivery of one chunk over T trees with one base and one enhancement layer.

The Unequal Error Protection allows depending on the selected code rates for base and enhancement layer, that the base layer can be still decoded if $l b$ trees are lost, whereas the enhancement layer is already lost at losing $l e$ trees, being $l b \geq l e$.

In case of using FEC as the error control method a Source Block (SB) has to be received to apply the FEC decoding and further allows for decoding the video. This results in an end-to-end playout delay, as depicted in Figure 6, which consists of the worst-case-delay, FEC decoding delay, which is the time needed to recover the original data from the FEC symbols, and the time needed by the client to receive the data of the SB from the different trees and start decoding the FEC. The latter delay is denoted as decoding delay. The decoding delay consists of the SB length in playout time $\left(t_{S B}\right)$ and an additional delay $\left(t_{\text {add }}\right)$, as shown in eq. (2), where $t_{S B}$ refers to the length of the SB in seconds, $B$ to peer's bandwidth (video rate + FEC rate) and $R$ to the video rate. The additional delay $t_{\text {add }}$ is the delay suffered at the beginning of the session, when the server waits for the SB becoming available from the SVC encoder in order to allow generating the redundancy packets and start sending the data with the transmission rate (media rate + FEC rate), as shown in Figure 6. Since the FEC is applied over a SB, which is formed by an entire number of GOPs, the SVC decoding delay (SVC dec delay) is already included in the decoding delay and therefore, it is not separately depicted in Figure 6. 


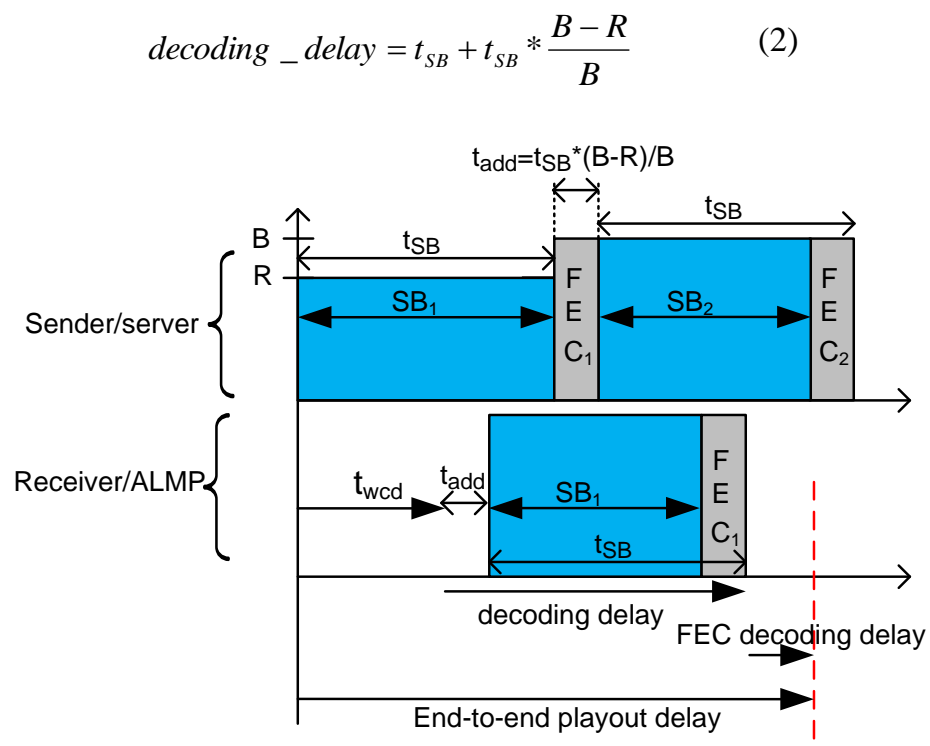

Figure 6: End-to-end playout delay for the FEC case

\subsection{SIMULATOR IN NS-2}

\section{SIMULATION}

A multi-tree ALM reference system with the features as described in section 2 and 3 has been implemented in the network simulator ns-2. At start of the simulation, a given number of nodes is initialized in the overlay network. Therefore, the nodes start a joining procedure by sending a message to the ALTM, which deals with the joining requests organizing the peers in the $\mathrm{P} 2 \mathrm{P}$ overlay. While participating in the streaming session, the nodes interchange messages with ALTM in order to update P2P structure and to establish new relationship when new peers join the service and others leave it.

The simulation time is divided in time intervals denoted as simulation_step. In each time interval, a random number of nodes disconnects for time $t_{-} O F F$. After $t_{-} O F F$, these disconnected nodes join the service again as new nodes, leading to a "stable" system, where the number of online nodes is approximately even over time. The number of nodes leaving the system in each simulation_step is calculated so that the percentage of nodes disconnected at any simulation time corresponds to a given peer_churn. This division of the time allows a more flexible and realistic simulation of the peer churn, since not all leaving nodes are forced to abandon the system at the same time instance, but the disconnections are distributed over time.

The media data is encapsulated in IP/UDP/RTP with multi-session transmission and a maximum packet size of 528 bytes at the streaming entity to be distributed among the different trees. The media distribution is carried out in a Round-Robin manner for each of the layers so that the data is equally distributed among the trees and the trees are of equal importance. For the FEC method, an emulated Raptor [13] decoding process is implemented based on the recommendations in [14]. An independent FEC fountain is applied to each layer at the root source, where the raptor encoding symbol size is varied in each source block (SB) to meet the requirements on symbols per SB in [14].

\subsection{SIMULATION SET UP}

The reference ALM system has been simulated in the ns- 2 test-bed with a number of nodes equal to 1000 over a $40 \mathrm{~s}$ streaming session. The RTT for the links between peers is randomly varied between 20 and $140 \mathrm{~ms}$. Overprovisioning rates for each peer are set to $[10 \%, 20 \%, 30 \%]$, this is the ratio of the additional bandwidth for error control (FF and FEC) over the video rate,. In order to analyze the impact of the overlay outages in terms of received quality, different peer churn rates have been simulated, as summarized in Table 1. 


\begin{tabular}{|c|c|}
\hline peer churn rates & $1 \%, 2.5 \%, 5 \%, 10 \%, 15 \%, 20 \%$ \\
\hline simulation_step & $100 \mathrm{~ms}$ \\
\hline$t \_O F F$ & $2000 \mathrm{~ms}$ \\
\hline
\end{tabular}

Table 1- Peer churn simulation parameters

In section 4.3, the two error control methods to overcome outages during ALM tree loss events and ALM overlay reconnection are evaluated. The methods FF and FEC are compared using SVC and AVC. For the simulation, a concatenation of four different ITU-T standard test sequences CITY, CREW, FOREMAN, SOCCER are encoded using SNR scalability with an extended constant bit rate encoding process which is based on the latest SVC reference encoder JSVM 9.17. The properties of the encoded streams are summarized in Table 2 and Table 3.

\begin{tabular}{|c|c|c|}
\cline { 2 - 3 } \multicolumn{1}{c|}{} & Video rate [kbps] & avg. PSNR [dB] \\
\hline AVC & 389 & 36.90 \\
\hline SVC- base layer & 149 & 31.80 \\
\hline SVC- base + enhancement layer & 391 & 36.08 \\
\hline
\end{tabular}

Table 2- Media test stream characteristics

\begin{tabular}{|c|c|}
\hline $\begin{array}{c}\text { Concatenated video } \\
\text { sequences }\end{array}$ & CITY,CREW,FOREMAN,SOCCER \\
\hline Coding mode & SNR (MGS) scalability \\
\hline Coding structure & IDR + GOP16 \\
\hline Resolution & QVGA \\
\hline Frame rate & $30 \mathrm{fps}$ \\
\hline
\end{tabular}

Table 3- General coding characteristics

For the FEC approach the SB length is set to $0.567 \mathrm{~s}$, which corresponds to an SVC GOP of size 16plus a preceding IDR frame (17 frames). In case using SVC, only the base layer is protected. For FF, the maximum number of retransmission requests per lost packet is fixed to 5 , which corresponds to the same tune-in delay as for the FEC case at an over-provisioning of 30\%. All error control settings are summarized in Table 4.

\begin{tabular}{|c|c|}
\hline \multicolumn{2}{|c|}{ Flow Forwarding (FF) } \\
\hline $\begin{array}{c}\text { Maximum number of } \\
\text { retransmissions }\end{array}$ & 5 \\
\hline Reserved bandwidth & $39 \mathrm{kbps}(10 \%), 78 \mathrm{kbps}(20 \%)$ and $117 \mathrm{kbps}(30 \%)$ \\
\hline pre-buffer & $0.567 \mathrm{~s}$ \\
\hline
\end{tabular}




\begin{tabular}{|c|c|}
\hline \multicolumn{2}{|c|}{ Forward Error Correction (FEC) } \\
\hline FEC code & Raptor \\
\hline Source block (SB) length & $0.567 \mathrm{~s}$ \\
\hline Code rate AVC & $0.9(10 \%), 0.83(20 \%)$ and $0.77(30 \%)$ \\
\hline Code rate base layer & $0.8(10 \%), 0.66(20 \%)$ and $0.57(30 \%)$ \\
\hline Code rate enhancement layer & 1 \\
\hline FEC coding delay & $0.567 \mathrm{~s}$ \\
\hline
\end{tabular}

Table 4- General ALM error control settings

Table 5 shows the number of consecutive retransmission requests that can be performed for the same packet keeping the delay equal to the end-to-end playout delay in case of using the FEC approach. The calculation has been carried out following the description of the delays in section 3.1 and 3.2 for a SB length of $0.567 \mathrm{~s}$, an average RTT equal to $80 \mathrm{~ms}$, a SVC dec delay of $82 \%$ of the SVC GOP time interval and a FEC decoding delay of $100 \mathrm{~ms}$.

\begin{tabular}{|c|c|}
\hline Over-provisioning (\%) & Retransmission requests \\
\hline 10 & $3-4$ \\
\hline 20 & 4 \\
\hline 30 & 5 \\
\hline
\end{tabular}

Table 5: Possible number of retransmission requests

\subsection{RESULTS}

In the following, the results are presented as obtained by the reference system test-bed. The results are shown in Figure 7, Figure 8 and Figure 9 with the naming format $X_{-} Y_{-} Z$, where $X$ refers to the over-provisioning factor, $\mathrm{Y}$ to the used of single (AVC) or scalable (SVC) coding and $\mathrm{Z}$ to the use of FF (RET) or FEC (FEC). In these figures, the average playout video quality is shown in terms of PSNR in $\mathrm{dB}$ and frame rate in terms of frames per second (fps) over different peer churn rates. PSNR and frame rate values are calculated as an average over all active peer nodes. If there is no video playout available at a peer, freeze-frame error concealment is used.

In Figure 7 for a over-provisioning rate of 10\%, SVC outperforms AVC for peer churn values higher than 2.5\% and $7 \%$ when using FF and FEC respectively. For higher peer churn values, gains up to $2 \mathrm{~dB}$ for FF and $4 \mathrm{~dB}$ for FEC can be reported compared to the use of AVC. The FEC also outperforms the FF approach for AVC in this case, since $10 \%$ over provisioning does not allow for all retransmission to be served by parent peers, thus peers with bad connection states saturate the system with retransmission requests.
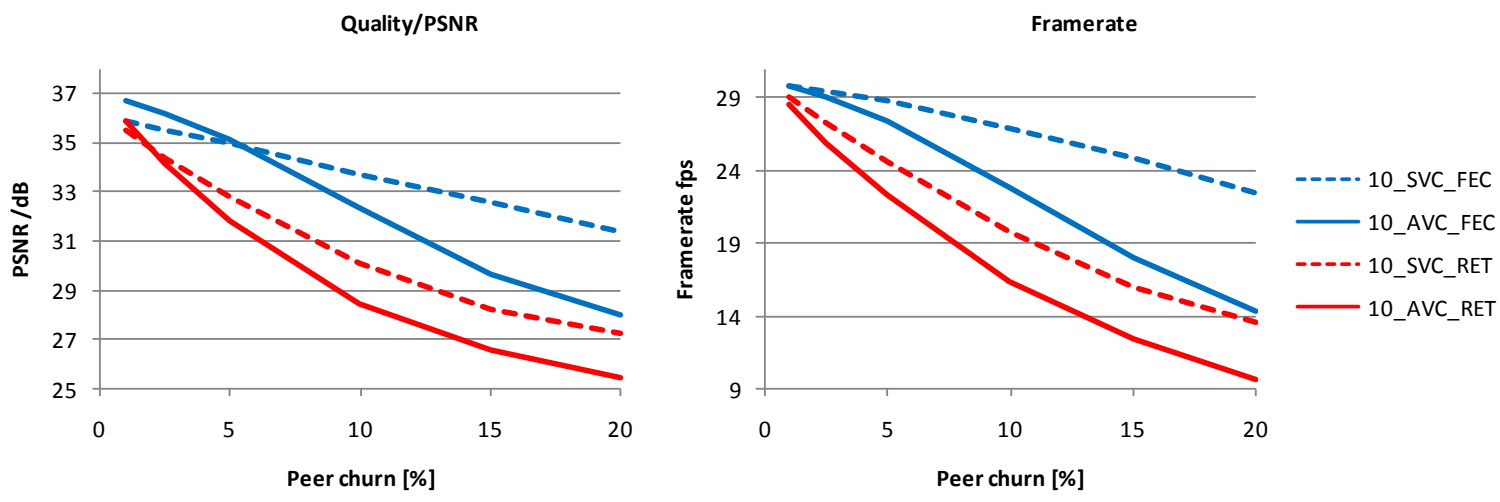

Figure 7- Average playout video quality for $10 \%$ over-provisioning: PSNR/dB and framerate 
For an over-provisioning rate of $20 \%$, there is a noticeable improvement in the video quality using FEC method for both AVC and SVC, as shown in Figure 8. The difference between single layer coding and layered coding is reduced, especially in the case of FEC. The coding efficiency penalty for SVC as shown in Table 2 is noticeable for peer churn rates up to $16 \%$. The FF method in combination with AVC performs less good for these overprovisioning values compared to SVC, for peer churn values higher than $8 \%$.
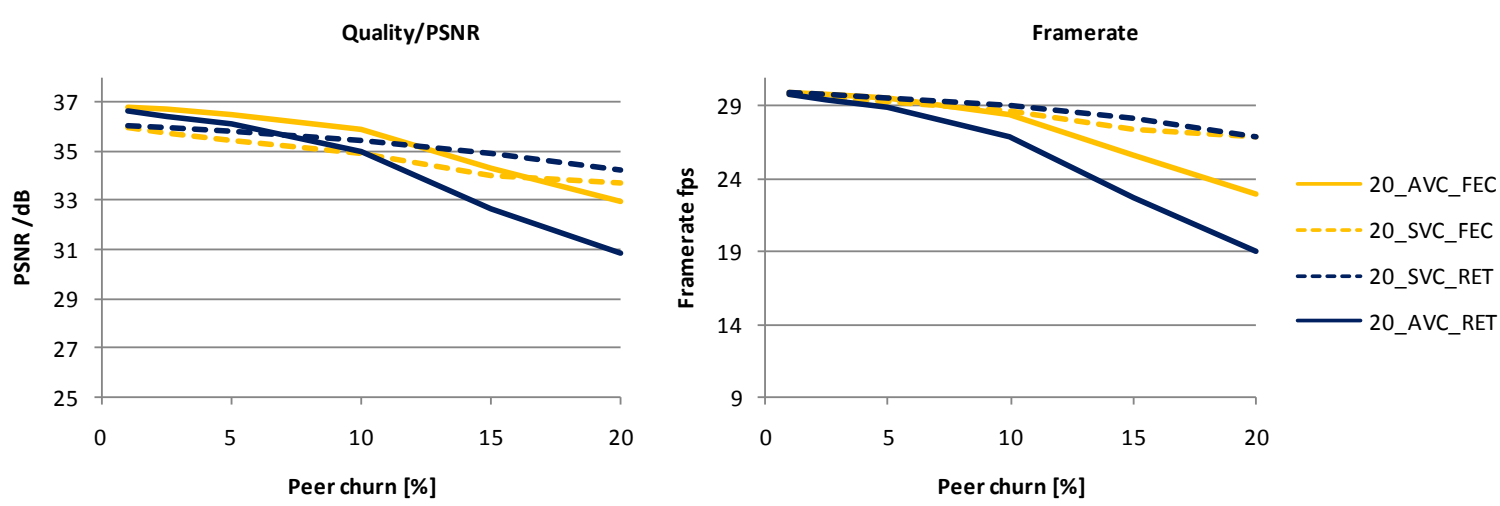

Figure 8- Average playout video quality for $20 \%$ over-provisioning: PSNR/dB and framerate

For an over-provisioning rate of $30 \%$, the SVC coding penalty dominates the gains of using SVC for a bigger range of peer churn rates. Up to churn rates of $10 \%$, AVC performs better for the case using FF as shown in Figure 9. When using the FEC approach, AVC outperforms SVC with respect to the received video quality.
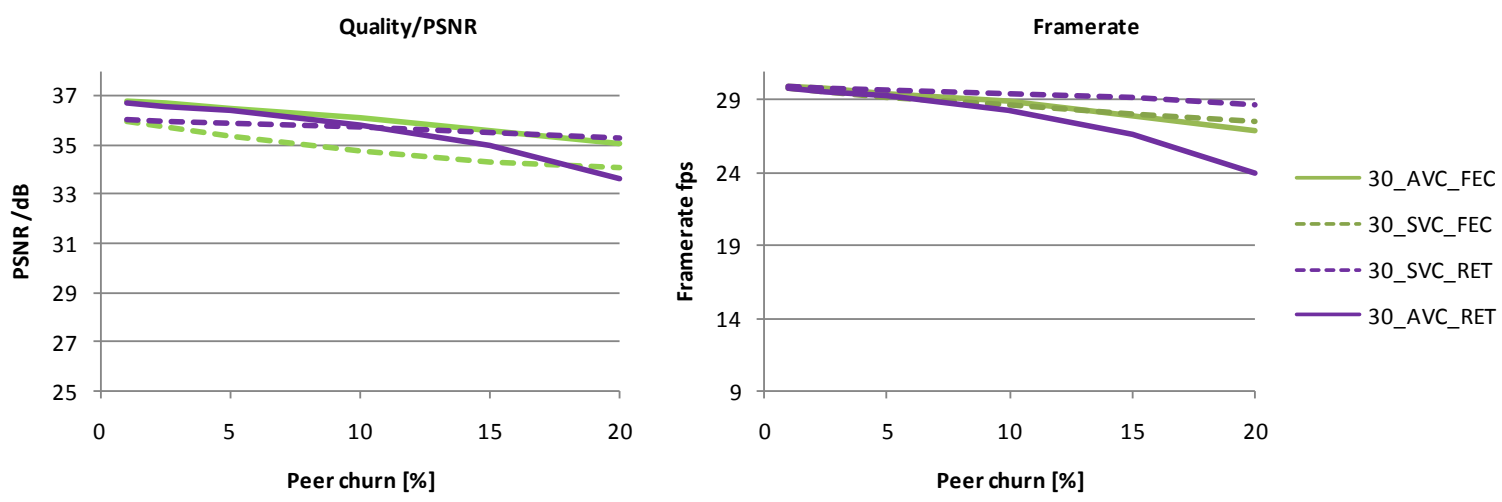

Figure 9- Average playout video quality for $30 \%$ over-provisioning: PSNR/dB and frame rate

The received video quality can be evaluated from a different perspective, i.e. by referring to the received and playable frame rate at the peers, as depicted in the right parts of Figure 7, Figure 8 and Figure 9. Here it can be noticed, that SVC outperforms AVC in all experiments, which leads to a different interpretation of the quality of the service, i.e. in the AVC cases although showing better video quality in terms of PSNR, a higher loss in terms of lost pictures is seen by the peers. Furthermore, a reasonable quality in terms of frame rate for the AVC cases is first achieved when higher over-provisioning rates (30\%) are used. Depending on the video sequences, there may be preferences of having a higher frame rate over higher PSNR values. Evaluating these cases lies outside the scope of this work but may be an interesting topic to look at. 


\section{CONCLUSION}

The benefits of using Scalable Video Coding (SVC) in combination with two different techniques for error control (flow forwarding and FEC) in live streaming over multi-tree Application Layer Multicast (ALM) are shown. The error control methods in combination with SVC clearly outperform the combination of error control with AVC for live streaming when overcoming connection outages during repair operations in multi tree-based ALM delivery systems, especially for low over-provisioning values up to $20 \%$. The advantage of relying on SNR scalability in comparison to single layer temporal scalability is presented based on the received quality in terms of PSNR and frame rate.

For up to $30 \%$ over-provisioning values, gains for using SVC are reported. It can be noticed that the SVC gain in combination with the presented error control methods is lost as the dedicated bandwidth for error control is increased. While the FEC approach consumes more bandwidth over all operation points of the system, the FF consumes less bandwidth but is sensitive to low uplink bandwidths at high peer churn rates. The received video quality with both approaches can be significantly improved using SVC. Especially in terms of playable frame rate at the peers SVC outperforms AVC in all experiments.

\section{REFERENCES}

[1] S. Deering, "Host extensions for IP multicasting", STD 5, RFC 1112, August 1989.

[2] J. Liu; S.G. Rao; B. Li; and H. Zhang, "Opportunities and Challenges of Peer-to-Peer Internet Video Broadcast", in proceedings of the IEEE, vol. 96, no. 1, pp 11-24, January 2008.

[3] Meng Zhang, Qian Zhang, Lifeng Sun, Shiqiang Yang, "Understanding the Power of Pull-Based Streaming Protocol: Can We Do Better?", in IEEE Journal on Selected Areas in Communications, vol. 25, no. 9, December 2007.

[4] V. N. Padmanabhan, H. J. Wang, and P. A. Chou, "Resilient peer-to-peer streaming ", in Proc. of ICNP, November 2003.

[5] M. Castro, P. Druschel, A.-M. Kermarrec, A. R. A. Nandi, and A. Singh, "SplitStream: High-bandwidth content distribution in a cooperative environment", in Proc. of ACM SOSP, October 2003.

[6] H. Schwarz, D. Marpe, and T. Wiegand, "Overview of the scalable video coding extension of the H.264/AVC standard", IEEE Trans. Circuits Syst. Video Technol., vol. 17, no. 9, September 2007.

[7] P. Baccichet, T. Schierl, T. Wiegand, and B. Girod, "Low-delay Peer-to-Peer Streaming using Scalable Video Coding", in Proc. of Packet Video Workshop, November 2007.

[8] G. Fairhurst and L. Wood (Eds.), "Advice to link designers on link Automatic Repeat reQuest (ARQ)", IETF RFC 3366, BCP 62, August 2002.

[9] J. Rey, D. Leon, A. Miyazaki, V. Varsa, and R. Hakenberg (Eds.), "RTP Retransmission Payload Format", IETF RFC 4588, July 2006.

[10]J. Ott, S. Wenger, N. Sato, C. Burmeister, and J. Rey (Eds.), "Extended RTP Profile for Real-time Transport Control Protocol (RTCP)-Based Feedback (RTP/AVPF)", IETF RFC 4585, July 2006.

[11]J. Noh, A. Mavlankar, P.Baccichet, and B. Girod, "Reducing End-to-End Transmission Delay in P2P streaming systems Using Multiple Trees with Moderate Outdegree", In Proc. of ICME, June 2008.

[12]M. Watson (Ed.), "Forward Error Correction (FEC) Framework", IETF FECFRAME working group, draft-ietf-fecframe-framework-05, July 2009.

[13]A. Shokrollahi, "Raptor Codes", in IEEE Transactions on Information Theory, vol. 52, no. 6, June 2006.

[14]3rd Generation Partnership Program (3GPP), "Multimedia multicast and broadcast service (MBMS); protocols and codecs (Rel. 6)", TS 26.346 V6.10.0, September 2007. 